\title{
Opportunity, Myth and Way: Research on E-commerce Live Women's Brand Communication Strategy Under the Long Tail Theory
}

\author{
Zhang Yu \\ School of Humanities and Communication, Zhejiang Gongshang University, Hangzhou, China
}

Email address:

2480977612@qq.com

\section{To cite this article:}

Zhang Yu. Opportunity, Myth and Way: Research on E-commerce Live Women's Brand Communication Strategy Under the Long Tail Theory. Science Innovation. Vol. 9, No. 3, 2021, pp. 118-123. doi: 10.11648/j.si.20210903.18

Received: March 26, 2021; Accepted: May 14, 2021; Published: May 24, 2021

\begin{abstract}
Research background: E-commerce Live broadcasting brings the most direct and convenient sales channel for women's clothing brands, greatly reduces the sales cost of businesses, and meets the prerequisite of The Long Tail Theory. Based on this, women's clothing brands communication strength rapidly improved, and the unpopular brands also got the opportunity to get out of the circle. Objective: From the perspective of The Long Tail Theory, this paper analyzed the long tail characteristics of women's clothing brands in e-commerce live broadcasting, analyzes the difficulties of women's clothing brand in e-commerce live broadcasting, and puts forward strategies to improve brand communication efficiency. Methods: This paper mainly used Participant Observation, virtual ethnography and case analysis methods to track more than 50 women's clothing live rooms on Taobao platform for more than 2 years, mainly observed the marketing methods, communication strategies and communication effects of major women's clothing brands. And participated in the purchase process of women's clothing brands, understood how women's clothing brands in e-commerce live broadcasting made the whole process from immersion sales to express logistics after-sales service. Results and conclusion: In this study, it was found that the women's clothing brands of e-commerce live broadcasting room meet The Long Tail effect and has the characteristics of long tail supply, long tail communication and long tail sales. But at the same time, there are also some problems in the transmission of women's clothing brands, so this paper puts forward the ways of women's clothing brands communication under The Long Tail theory.
\end{abstract}

Keywords: The Long Tail Theory, E-commerce Live Broadcasting, Women's Brand, The Difficultie, Communication Strategy

\section{机遇·迷思·进路：长尾理论下电商直播女装品牌传播策略}

\section{张玉}

浙江工商大学人文与传播学院, 杭州, 中国

\section{邮箱}

2480977612@qq.com

\author{
摘要：研究背景：电商直播为女装品牌带来最直接、最便捷的销售渠道，极大的降低了商家的销售成本，满足了长尾 \\ 理论的先决条件。在此基础上，女装品牌传播力度急速提升，冷门小众的品牌也获得了出圈机会。目的：本文就从长 \\ 尾理论的视角出发, 分析电商直播女装品牌的长尾特性, 并分析女装品牌在电商直播中存在的困境, 提出提升品牌传 \\ 播效益的策略。方法: 本文主要使用参与式观察法、虚拟民族志以及案例分析法对 50 多个淘宝平台女装直播间进行跟 \\ 踪研究, 时间长达 2 年以上, 主要观察各大女装品牌的营销方式、传播策略以及传播效果。并参与女装品牌的购买过程, \\ 了解电商直播中女装品牌从沉浸式销售到快递物流售后服务的全过程。结果和结论：在研究中发现，电商直播中女装
}


品牌满足长尾效应, 具有供给长尾、传播长尾、销售长尾的特点。但同时, 电商直播在女装品牌传播中也存在一些问 题，因此本文从这些问题出发，提出长尾理论下电商直播女装品牌传播的进路。

关键词：长尾理论，电商直播，女装品牌，困境，传播策略

\section{1. 引言}

\section{1. 研究背景}

女装品牌是中国电商直播产品的主要类目, 占所有电 商直播行业总产值的一半以上。根据新榜研究院数据显示: 2019年，服饰类的商品构成占电商直播总商品的 $46 \%$, 是 直播带货中交易额最大的品类; 2020年上半年，服装服饰 直播商品数 854.9 万, 在直播电商全品类中占比 $37.6 \%$; 直 播间服装鞋帽、针纺织品类商品上架超 1.5 亿次, 上架次 数占比 $41.7 \%, \mathrm{SKU}$ 占比 $53.2 \%$ 。目前, 服饰已成为淘宝、 抖音、快手等主流直播电商平台的重点销售品类之一。但 不可否认, 女装品牌在电商直播过程中也存在一些困境, 电商直播的快速发展也给我们带来很多迷思, 因此在此基 础上, 探索女装品牌传播进路及其重要。

\section{2. 研究目的和意义}

电商直播为女装品牌传播提供了最佳传播渠道, 众多 不知名女装品牌在电商直播环境中形成自己的品牌声浪, 提升了传播效益。在研究过程中发现, 女装品牌在电商直 播中满足长尾理论的先决条件。因此以长尾理论的视角出 发, 探索女装品牌在电商直播过程中存在的长尾特性, 探 究目前电商直播在女装品牌传播过程中的作用, 以及存在 的诸多困境, 可以提升女装品牌营销的精准性, 为女装品 牌传播提供一定的思考。

\section{3. 理论价值和实践价值}

\subsection{1. 理论价值}

电商直播近年来发展迅速, 在产生巨大经济效益的 同时, 也为理论研究提供了众多视角。但目前电商直播 与女装品牌传播结合的研究还处于空白, 本文就从“长尾 理论” 的视角出发, 探究电商直播在女装品牌传播中的 “机遇”“迷思”和“进路”。一方面充实电商直播与女装品牌 传播结合的研究, 另一方面为“长尾理论”提供新的研究 视角。

\subsection{2. 实践价值}

电商直播的兴起为各类品牌传播提供了新的场域, 女 装品牌更是成为最大受益方。就淘宝直播平台而言, 目前 女装品牌已经成为第一销售类目。因此从长尾理论的视角 研究电商直播下女装品牌传播的策略具有很强的现实意 义。首先, 可以发现电商直播中女装品牌传播的问题, 引 发反思; 其次, 探究传播策略可以促进女装品牌的精准营 销; 此外, 可以为其他品牌在电商直播中的传播提供一定 的借鉴意义。

\section{4. 相关领域目前的研究进展}

电商直播具有极强的交互性, 为新时代的场景营销提 供了最佳路径。学者简承滟, 冯思潮 (2020) 认为电商直 播在实现用户留存、调动用户购买意愿方面蕴藏着巨大的 营销潜力, 但同时也存在很多乱像[1]。学者陈瑶（2020） 从景观社会的视角出发, 探索目前电商直播“直播+”的跨界 发展模式, 认为电商直播是令人目眩神迷的“景观秀”[2]。 王林玉, 李莉莉, 陈洁等 (2014) 学者从客户体验的视角 出发, 探究了服装电商企业产品营销策略, 提出电商企业 可以利用视频生动的呈现服装效果, 缩短消费者的认知时 间, 为消费者带来多元体验[3]。学者裴学亮邓辉梅 (2020) 基于淘宝电商直播的购物过程, 以“人-商品-场景” 3 个要素 出发, 探究了电商直播情境中产生的问题, 认为在电子商 务平台中直播电商在价值共创行为过程中需要主动提出价 值主张、关注产品互动和媒介互动[4]。孟昭宇（2017）研 究了跨境电商群直播商业模式下的品牌营销策略, 认为现 场直播时的受众互动、策划内容的精心设计、跨境货品的 高品质, 让跨境电商的专业化直播运营能够造就更高转化 率[5]。李莉莉（2014）探究了品牌服装电商企业在多品牌 运营、平价策略、渠道策略等方面的利弊, 认为企业应该 从提升品牌形象内涵、打造一流设计团队, 注重个性与体 验营销以及建立线上十线下的多元营销理念来提升品牌电 商运营效益[6]。丁蓄、刘瑜（2017）以女装品牌“MG小象” 为例, 探究了该品牌在淘宝电商直播中的直播效果, 认为 该品牌连续直播 12 小时、主播与粉丝的互动率高以及满足 粉丝多层次需求方面发挥了最大的优势, 因此品牌传播效 果良好[7]。

Shi Y（2020）认为电子商务流媒体直播中, 消费者 容易产生羊群效应, 从而影响其购买意愿。感知易用性、 情境因素和跟随他人行为对购买意向有显著影响, 感知有 用性对购买意向的影响较小, 不完全信息不会直接影响消 费者的购买意向, 但跟着别人看可能会影响他们看直播时 的购买态度 [8]。A Wongkitrungrueng, $\mathrm{N}$ Assarut, JO Business等 (2020) 学者从流媒体直播在建立消费者信任 和与社会商业卖家互动中的作用方面进行了探究, 认为符 号价值通过对销售者的信任对顾客参与有直接和间接的 影响, 而功利价值和享乐价值通过顾客对产品的信任和对 销售者的信任间接影响顾客参与[9]。Geng R, Wang S, Chen $\mathrm{X}$ 等学者从流媒体直播出发, 认为网络名人代言已经 成为电子商务卖家普遍的营销方式, 因此从理论和实践两 方面探讨网络名人代言对电子商务环境下营销效果的一 阶效应和二阶效应 $[10]$ 。

综上所述, 目前国内外学者对电商直播的研究大多集中 在现象解读、商家与用户关系、平台营销便利性以及电商参 与者之间的关系为主。仅有小部分学者以个别服装品牌举例 探究电商平台对服装营销的便利性, 鲜少有文献专门研究电 
商直播与女装品牌营销的关系。因此本文就从该视角入手, 探索长尾理论下电商直播下女装品牌的传播策略。

\section{2. 长尾理论和电商直播概述}

\section{1. 长尾理论定义}

“长尾理论”是由克里斯·安德森(Chris Anderson)在 2004年提出, 该理论主要考虑商品的成本和效率因素。认 为只要商品的存储、流通、展示的场地和渠道足够宽广, 并且销售成本急剧降低时, 即使再冷门的商品, 只要有人 卖, 就会有人买 [11]。这一理论和传统“二八定律”相违背, 即 $20 \%$ 的核心产品创造 $80 \%$ 的效益。克里斯·安德森通过研 究网上书城的盈利模式后发现, 产生利润的并不是所谓的 热门产品, 反而是来自于那条长长的尾巴, 也就是冷门产 品和多重用户评价 $[12]$ 。

因此从以上理论可以得出, 当尾部品牌拥有便捷效率 的流通渠道以及足够低的销售成本时, 其销售能力依然可 以与头部产品抗衡。而电商直播就为女装品牌的流通提供
了非常便捷的渠道, 且每个人都可以做主播, 将自己的小 品牌服装进行展示出售, 极大的降低了销售成本, 因此文 章将从这一视角出发, 探讨长尾理论下电商直播女装品牌 的传播策略。

\section{2. 电商直播}

电商直播是一种新型的购物方式, 主要利用直播技术 手段, 通过主播对产品特点以及优势进行讲解, 使得消费 者能够实时观看, 并进行线上下单购买的消费模式[13]。 电商直播自产生以来, 极大地带动了线上产品销售额, 降 低了销售成本、购物成本，并为商家提供了及其便利的收 货渠道[14], 做到了随时随地可直播、可收货。从2020年 12月份的淘宝直播行业流量数据来看（下图1），时髦穿 搭行业的流量数据稳居第一, 达到 1.47 亿, 占总行业的 $56.90 \%$ 。从时髦穿搭行业直播场次与观看比例来看, 直播 场次与观看次数基本持衡, 甚至某些时刻直播观看次数远 远超过直播场次, 这就说明时髦行业直播带货远远没有达 到饱和, 用户观看需求大于直播品牌直播供给（图2）。

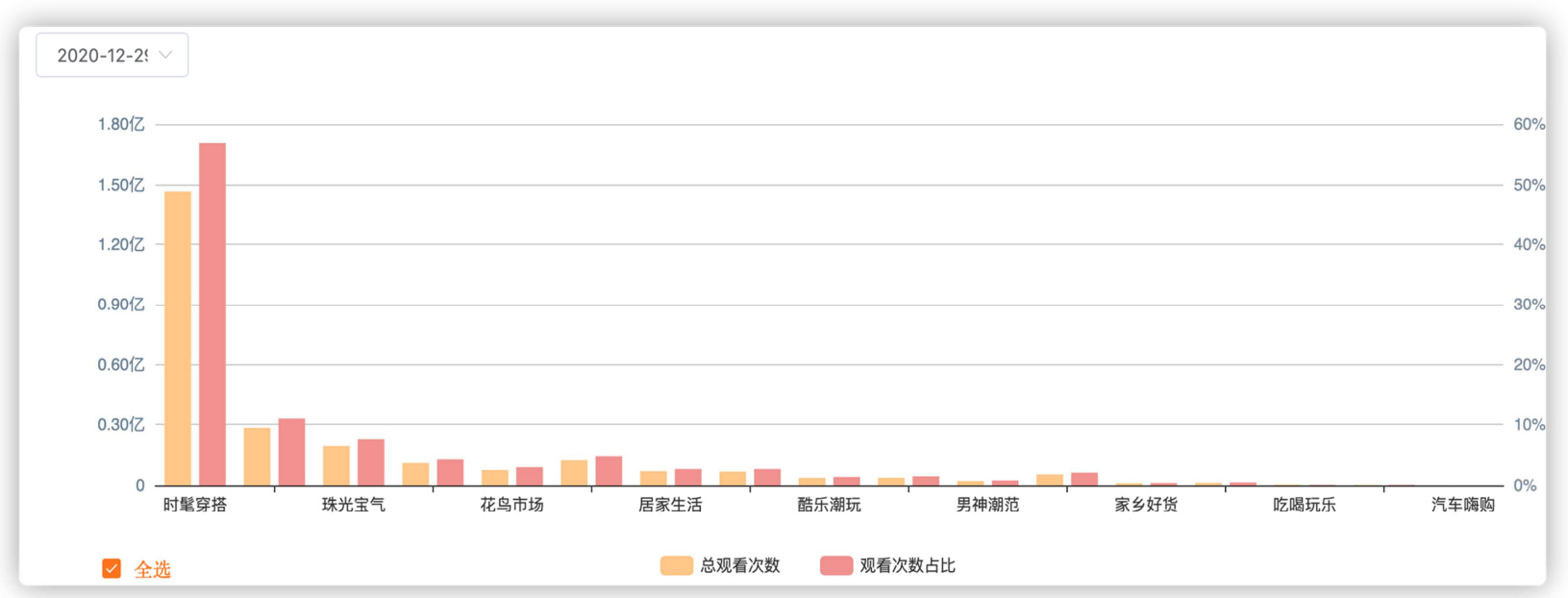

图1 2020.12.01-2020.12.29日淘宝直播行业流量趋势图(数据来源：知瓜数据)。

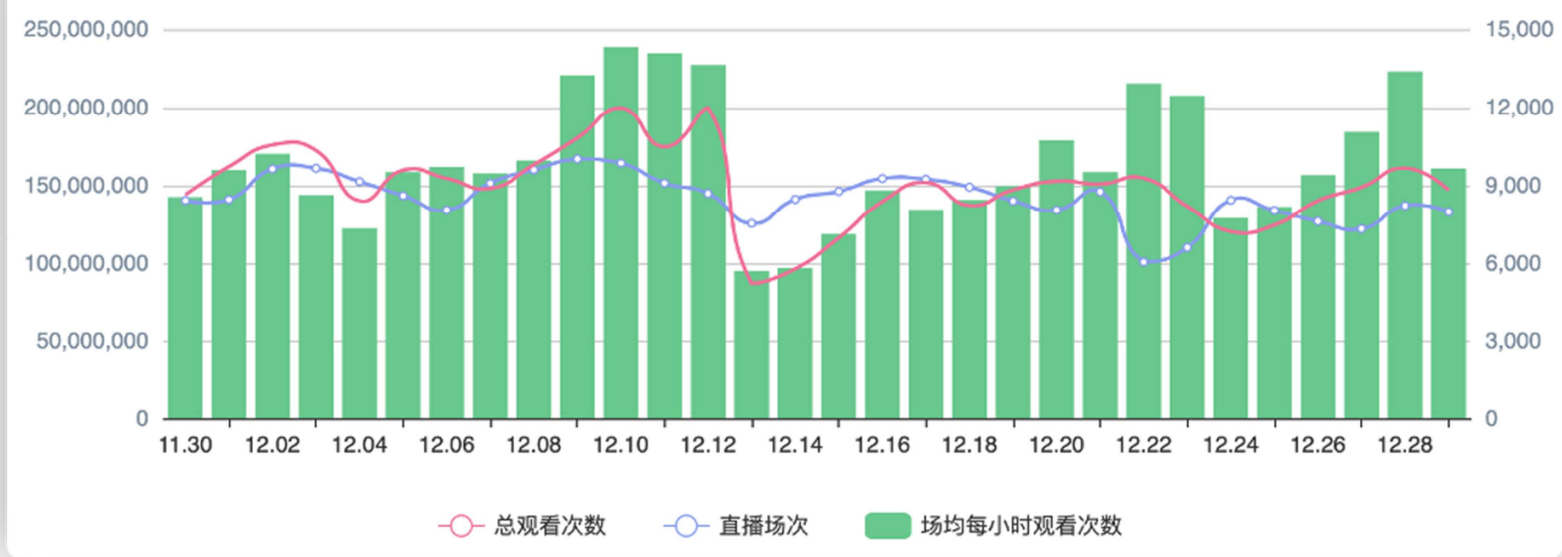

图2 2020.11.30-2020.12.28日淘宝直播时髦穿搭行业直播场次与观看比例(数据来源: 知瓜数据)。 


\section{3. 机遇:长尾效应在电商直播女装品牌传播中的 应用}

\section{1. 供给长尾:个人品牌催生差异化需求}

通过电商直播带货这一窗口, 许多女装类小众品牌重 获新生, 独特的女装品牌设计可以展现独一无二的自我, 许多冷门的女装品牌成为新一代女性青睐的爆款。而正是 由于电商直播对小众品牌的促进作用, 又鼓励了众多个人 商家不断涌入直播行列, 从而促使了小众女装品牌的繁荣, 使得小众女装品牌的供给提升, 各类风格的小众服装品牌 均有生存空间，供女性消费者挑选，小众品牌的独特创新 的设计思路不断催生女性消费者差异化的需求, 从而达到 了供给长尾。

\section{2. 传播长尾: 电商平台成为小众品牌聚集地}

电商直播自2016年上线以来，先通过淘宝、快手平台 进行测试, 取得良好的经济效益。从2018年开始, 大量新 兴平台均进行大量量投资, 加速布局电商直播产业, 并在 2020年上半年突破3000亿元产值（图3）。其主要原因就 是电商直播售货存储、流通、展示的场地和渠道的便利性, 促使品牌商和消费者快速、直接达成交易，且销售成本非 常低。因此不断聚集了大中小企业以及个体户入驻，成为 品牌聚集地, 尤其是小众品牌的潜力得到了前所未有的释 放窗口。这些特点正好满足了长尾理论的两个重点要素, 因此形成了传播长尾。

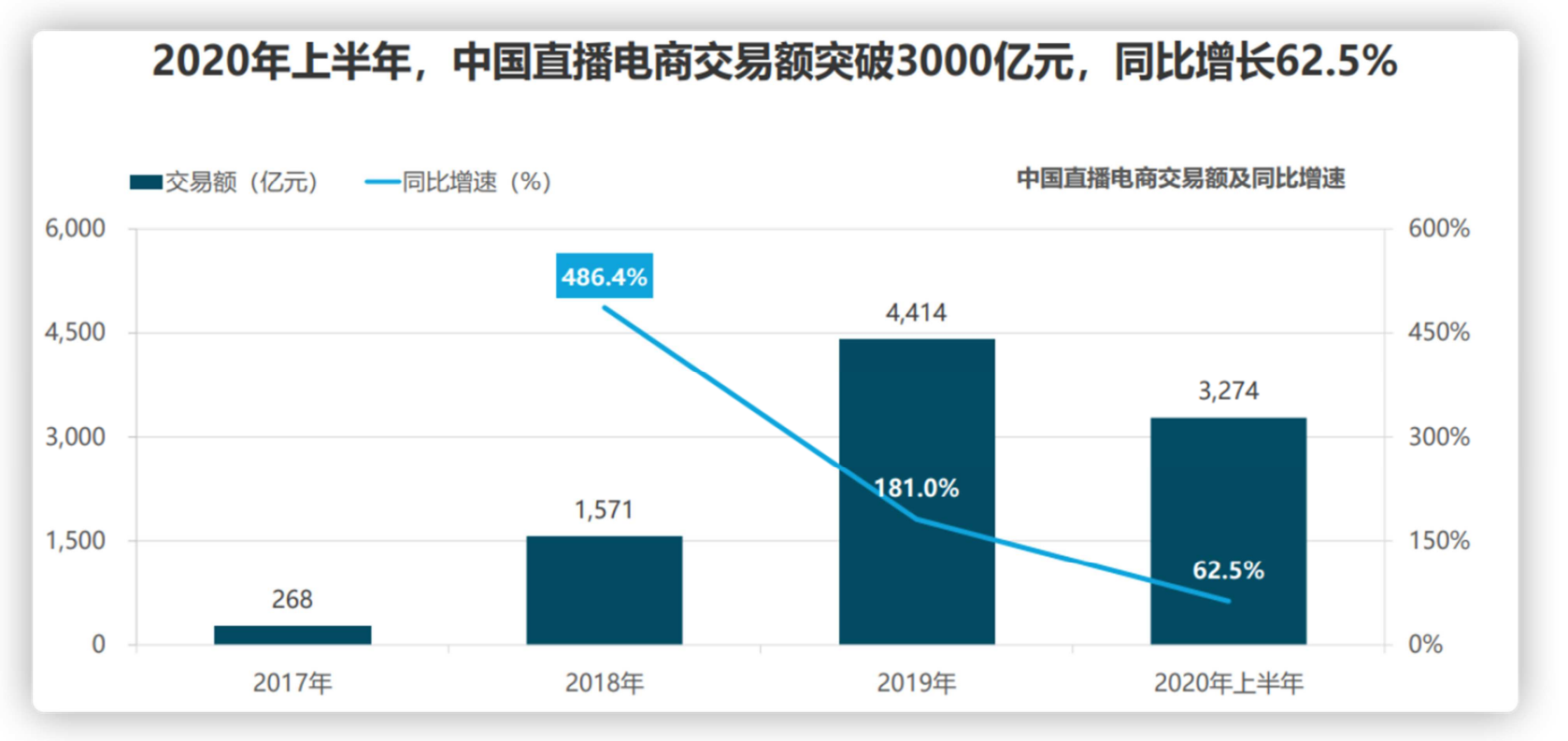

图3 2017-2020年中国电商直播交易额（数据来源：fastdata极数）。

\section{3. 消费长尾:便捷搜索引擎下的多元选择权}

目前，电商直播领域进行了垂直细分，就女装品牌而 言, 其子类品牌众多, 且分类详细, 再小的需求也适配合 适的供给。在精准化、标签化、个性化搜索的前提下，通 过丰富便捷的的搜索引擎，消费者拥有多元选择权。往往 直播产品在个人品牌微店中都有陈列, 且同样的服装还可 以通过“搜索相似产品”来进行对比竞价, 消费者的选择权 大大提升。同时, 在便捷的搜索与算法推荐机制下, 再冷 门的产品都拥有较大的销售空间。

\section{4. 迷思:长尾理论下电商直播女装品牌传播困境}

\section{1. 小众品牌粉丝群体粘性较低}

综合目前女装品牌电商直播的销售情况，女装品牌面 临的最大问题就是粉丝群体粘性较低。因为电商直播售货 一般是实时直播, 且直播时长至少长达 $3-5$ 小时, 因此同 一粉丝很难坚持看完全程直播。直播画面中的主播要坚持
时时在场, 画面不能长时间留白, 否则粉丝就会因为长时 间的等待退出直播间。如果主播离开画面超过 5 分钟, 观 看的粉丝很容易转移注意力。此外, 由于粉丝的粘性需要 长期培养, 因此对于小众品牌直播而言难度更大, 小众品 牌方无法做到每天上新新品服装, 没有新品就无法长期满 足粉丝的新奇心里, 很难吸引粉丝到场, 造成粉丝的流动 性较大, 粘性持久度较低。

\section{2. 众人进场, 瓜分电商红利}

由于电商直播成为近几年的最火爆的销售方式，吸引 了众多资本、众多大品牌入场, 形成了众人瓜分电商红利 的局势。尤其是 2020 年因为疫情困境, 电商直播再一次成 功出圈, 在疫情期间电商直播带货取得非常可观的业绩。 相比于其他销售渠道, 电商直播成为疫情期间不可替代的 销售平台, 且为中国复工复产做出了巨大贡献。借助电商 直播的火爆之势, 众多品牌纷纷进场, 进行销售场地的转 移, 进而瓜分电商红利。可以发现, 2020-2021年, 众多 明星带货入场, 分割了众多流量。从下图4可以看出, 疫 
情发生之后各大平台电商直播交易额出现火速蚛涨。如: 刘涛、胡可、谢娜、蔡少芬等明星纷纷加入直播行列，因 为女明星自带意见领袖效应, 因此电商直播红利被再次瓜 分。就女装品牌而言，其本身竞争非常激烈，品牌的更新
换代速度也非常快，因此同各类头部主播、明星主播互相 竞争，小众女装品牌的长尾效应被削弱，在激烈的电商直 播市场竞争中容易处于劣势。

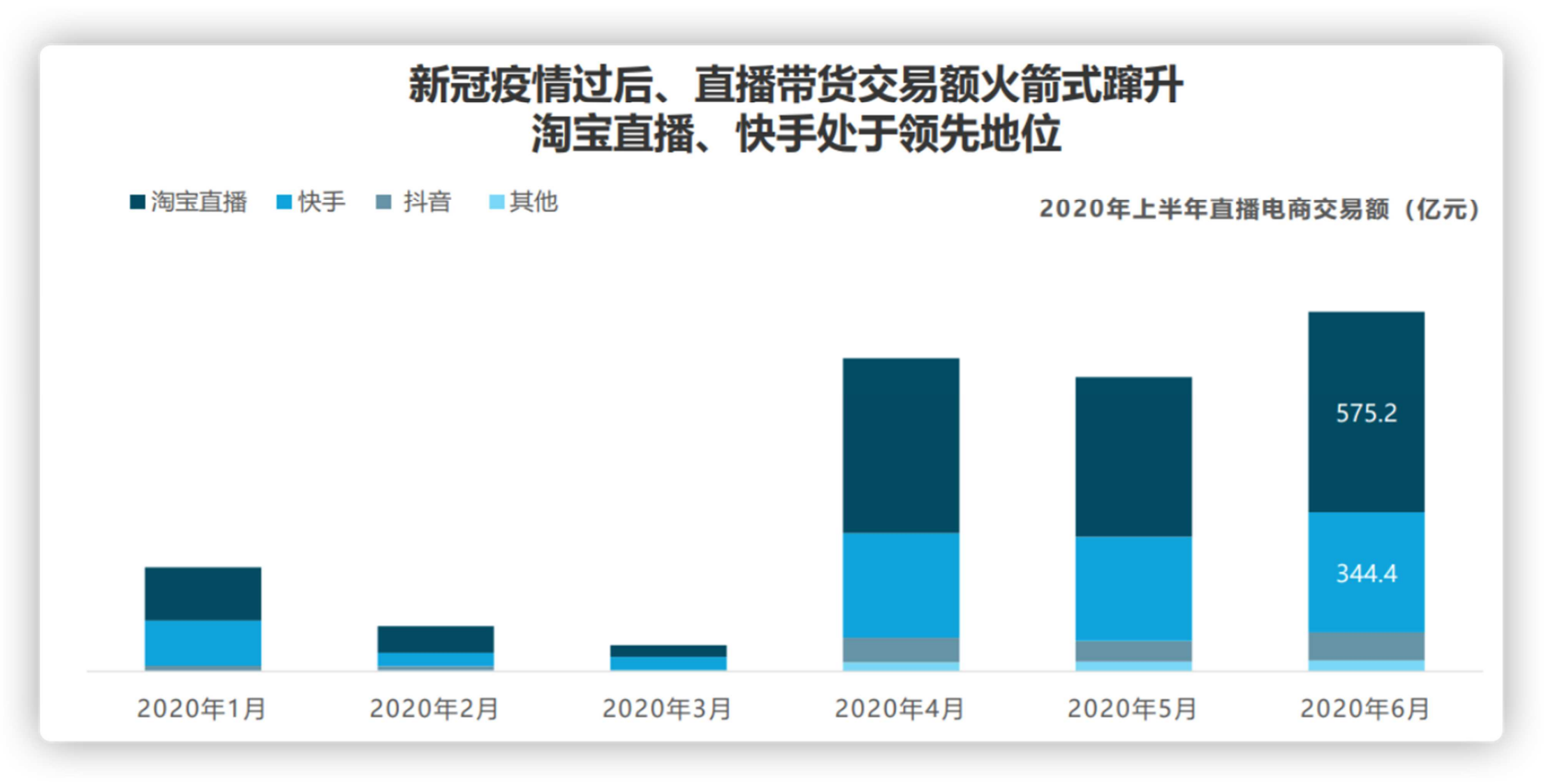

图4 疫情期间中国电商直播交易额增长图（数据来源：fastdata极数）。

\section{3. 粉丝注意力稀缺, 处于选择迷雾中}

杨国斌教授在《连线力》一书中提到: “新媒体既是 因也是果, 许多问题既是新媒体带来的, 却也是造成新媒 体现象本身的原因”[15]。电商直播的快速走红印证了这句 话, 伴随着各种直播平台的兴起, 粉丝的注意力被不断分 割, 尤其女装品牌的电商直播模式更是对粉丝注意力的疯 狂掠夺。粉丝在场的数量直接决定了女装品牌直播商家的 交易额。从几位女装品牌（陈洁kiki、雪梨CCherie、组艾 妈私服定制等) 头部主播来看, 其每晚的直播在场粉丝数 量从200-800万人不等。因此各大女装品牌主播共同争夺 同一批女装品牌粉丝的注意力, 使得直播粉丝的选择权大 大增加, 在眼花缭乱的主播直播中, 粉丝陷入选择迷雾, 在各大直播间来回跳转。这种困境也增加了粉丝对同一家 女装品牌的忠诚度, 使得女装品牌的长尾效应降低。

\section{5. 进路:长尾理论下电商直播女装品牌传播路径 探索}

\section{1. 精准挖掘粉丝需求, 进行超前需求供给}

面对小众品牌直播粉丝持久度较低的问题, 还是需从 挖掘用户需求方面攻破。女装品牌追求流行趋势, 追求当 季新款与超前爆款打造, 因此小众品牌依然可以挖掘粉丝 超前需求, 进行服装款式更新。例如: 在秋季末期, 一般 各大直播间会上新冬季派克服以及名贵的貂类女装; 在每 年12月份会上春季新款。这种超前供给的方式可以直接提 前收割粉丝的冬季需求, 满足后期粉丝需求, 同时在竞争
中挤压掉其他新款更新速度慢的品牌。另外, 目前各大女 装直播间一般直播流程为: 开播优惠款秒杀、新款上新、 隔天新款展示等环节。在隔天新款展示中, 一般女装直播 博主会在直播间和粉丝进行线上实时互动, 介绍隔天新款 的同时直接询问粉丝喜不喜欢展示的服装。通过这种最直 接的方式了解用户喜欢什么、需要什么, 获得用户反馈。 并根据粉丝反馈决定隔天的新品会不会上新, 这样一来既 保证了上新款一定有人购买, 又保证了和粉丝的互动效果, 还提升了粉丝粘性。因为如果粉丝有喜欢的新款, 第二天 很大程度会来购买, 隔天的粉丝在场数量也会提升, 达成 三赢的效果, 也提升了品牌的供给长尾。

\section{2. 优化服装设计, 提升品牌核心竞争力}

面对女装直播品牌竞争力非常激烈的问题, 最主要的 方式还是从品牌质量抓起, 进行服装设计优化, 提升品牌 的核心竞争力, 开发长尾产品。例如在服装制作前期, 把 控好服装材料的进货渠道, 挑选优良的服装材质; 在服装 设计过程中, 要秉持原创的原则, 进行更加人性化的设计, 而不是一味的追求潮流, 忽略服装的实用性; 在服装上新 过程中, 也要进行合理定价, 并为直播粉丝全面展示服装 的优缺点, 而不是只讲优点, 避谈缺点, 有效避免粉丝购 买后的落差感, 降低买家款和卖家款之间的落差。此外, 在直播售后服务中, 要和物流公司进行高效对接, 提升送 货物流速度, 在退货、换货等处理中要服务周到, 给粉丝 预留好感, 提升回购率。综合来看, 服装品牌的核心竞争 力的提升需要在各个环节进行细节把控, 但是最主要的还 是服装品质, 品牌服装品质好才能提升粉丝的长尾效应, 
让粉丝形成品牌记忆, 提升品牌的市场传播音浪, 减少购 物的退货率。

\section{3. 进行产品专场直播, 降低粉丝时间成本}

面对粉丝注意力稀缺的问题，可以从分割粉丝时间， 提升粉丝时间利用效率入手。将女装品牌进行垂直细分, 并进行专场直播预告, 让粉丝了解在某天某个时间段可以 买到自己需求的款式, 进而达成品牌直播和粉丝到场的心 理契约。可以解决粉丝注意力稀缺, 处于选择迷雾等的问 题。例如以服装种类进行专场设置。羽线服专场、派克专 场、春季专场、以及鞋包专场、毛衣内搭专场等。在这种 细分中, 粉丝可根据自己的需要和时间决定是否观看直播, 并且到场的粉丝一定会买到自己需要的服装品类, 提升了 粉丝购物满足感。以节令节日等时间作为专场设置, 双 11 钜惠专场、元旦专场、跨年夜专场、女神节专场、春节专 场等。在特定的节日以促销的方式吸引粉丝到场, 并收获 了粉丝闲暇时间的注意力, 提升了品牌的销售长尾。

\section{6. 结论}

综合以上, 在长尾理论视域下电商直播女装品牌具有 供给长尾、传播长尾、消费长尾的特性。在这种背景下, 大小女装品牌都有可能获得良好的经济效益, 尤其小众品 牌也可能与大品牌抗衡。但不可否认, 目前女装品牌在直 播过程中也遇到小众品牌粉丝群体粘性持久度较低、众人 进场, 瓜分电商红利、粉丝注意力稀缺, 处于选择迷雾中 等的问题, 面对这些问题, 品牌直播商家可以从精准挖掘 粉丝需求, 进行超前需求供给; 优化服装设计, 提升品牌 核心竞争力; 进行产品专场直播, 降低粉丝时间成本方面 着力, 进而提升女装品牌的传播效益。

\section{参考文献}

[1] 简承浑, 冯思潮. 电商直播:站上风口, 更要掌握航向[J]. 新 闻战线, 2020(15):49-51.

[2] 陈瑶. 景观社会视域下的电商直播 [J]. 青年记者, 2020, No.670(14):23-24.
[3] 王林玉，李莉莉，陈洁,等. 基于客户体验的服装电商企业 产品营销策略研究 [J]. 纺织导报, 2014(11):88-91.

[4] 裴学亮, 邓辉梅. 基于淘宝直播的电子商务平台直播电商 价值共创行为过程研究 [J]. 管理学报, 2020, v.17;No.170(11):56-65+120.

[5] 孟昭宇. 跨境电商群直播商业模式下品牌营销策略 [J]. 对 外经贸实务, 2017, 000(005):59-62.

[6] 李莉莉. 品牌服装电商企业运营与营销策略浅谈——以韩 都衣舍为例 $[J]$. 纺织导报, 2014(8):82-83.

[7] 丁蓄, 刘瑜. 淘宝直播在电商服装品牌中的应用分析以淘宝女装 "MG 小象"为例 $[\mathrm{J}]$. 戏剧之家, 2017(24):228-229.

[8] Shi Y. A Study on the Influence of E-commerce Live Streaming on Consumer's Purchase Intentions in Mobile Internet[C]// International Conference on Human-Computer Interaction. Springer, Cham, 2020.

[9] Wongkitrungrueng A, Assarut N, Business J O, et al. The role of live streaming in building consumer trust and engagement with social commerce sellers[J]. 2020.

[10] Geng R, Wang S, Chen $\mathrm{X}$, et al. Content marketing in e-commerce platforms in the internet celebrity economy $[\mathrm{J}]$. Industrial Management \& Data Systems, 2020, ahead-of-print(ahead-of-print).

[11] 黄孝章、张志林、刘双双《长尾理论促进新媒体经济增长 方式的转变》, 《集团经济研究》2007年第12期。

[12] 韩晓飞. 长尾理论视域下内容型网红价值变现研究—— 美食博主"李子染"为例[J]. 硅谷, 2019, 005(012):95-97.

[13] 王馨. "直播+"时代下服装电商营销策略创新[J]. 经营与管 理, 2017, 000(011):118-120.

[14] 顾钰炜. 电商直播对消费者购买决策影响研究 [J]. 价格理 论与实践, 2020, No.428(02):126-129.

[15] 杨国斌. 连线力: 中国网民在行动 $[\mathrm{M}]$. 广西师范大学出版 社, 2013. 